\title{
Analysis of Influence on Quality of Life of Liver Cancer Patients of Health Education Based on WeChat Public Platform
}

\author{
Ruixin Lin ${ }^{1}$, Shuli Yang, Yan Jia, \\ Wenwen Jian, Qing Yu, Min Wang and Limei Fan ${ }^{{ }^{*}}$ \\ ${ }^{1}$ The Second Hospital of Jilin University, Changchun city Jilin Province, 130012 \\ adggfjg@126.com
}

Keywords: WeChat public platform, Health education, Liver cancer, Quality of life

\begin{abstract}
Objective: The health education of liver cancer patients on WeChat public platform is conducted, and the correlation between liver cancer and the quality of life, anxiety and depression is observed and analyzed. Method: In our hospital, 88 cases of liver cancer patients are selected as observation objects. They are randomly divided into a control group and a treatment group by random numbers. The control group is performed general health education in general hospitals, and the treatment group is conducted health education on WeChat public platform. According to HAMD, SAS and QOL, the evaluation is conducted to analyze the differences between the patients of the two groups and evaluate the improvement of the depression, anxiety symptoms and quality of life of them. Result: Compared with the control group, SAS score of the patients in the treatment group is significantly reduced, and the difference between the groups is significant $(\mathrm{P}<0.05)$. Compared with the control group, the anxiety score of the patients in the treatment group decrease significantly, and there is a significant difference between groups $(\mathrm{P}<0.05)$. After the health education, the quality of life score of the two groups is significantly improved compared with the control group $(\mathrm{P}<0.05)$. Conclusion: The health education of WeChat public platform has obvious improvement effects on patients with liver cancer, reducing the degree of anxiety and depression, improving the patients' psychological state and the quality of life, and promoting the early recovery, which has a very important clinical significance.
\end{abstract}

As the sixth most common malignant tumor in the world, liver cancer has the characteristics of rapid onset, rapid change of disease, and severe illness, affecting the lives and work of patients, It requires early diagnosis and timely adoption of effective treatment measures. The pathogenesis is more complex, and the main cause may be related to viral cirrhosis, liver cirrhosis, carcinogens and other aflatoxin and environmental factors ${ }^{[1]}$. Liver cancer has great threat to patients' lives if they do not receive surgery in time. Liver cancer belongs to the high incidence of tumors in our country. In China, liver cancer patients account for at least half of the world's total liver cancer patients, which seriously endangers health. At present, the treatment of liver cancer mainly adopts surgical treatment methods, most of which are liver resections, and then there are problems in liver function after surgery. The implementation of the nursing model in the new situation is mainly centered on patients and human health rather than on the disease. The patients' health care awareness and the demands for health knowledge are increasingly strong ${ }^{[2-3]}$. Caregivers need to take effective health education measures to increase patients' awareness of disease and health and actively improve quality of life after surgery. Intervention with health education is an inevitable trend in the development of clinical work ${ }^{[4]}$. However, as the society progresses and people's living standards continue to increase, information exchange focuses more on interaction and effectively changes people's communication and communication methods. For example, the adoption of WeChat public platform is the current trend. Therefore, this paper carries out health education on WeChat public platform for liver cancer patients and examines the changes in the patients' quality of life.

\section{Data and Method}

\section{Data}

Eighty-eight cases of liver cancer patients who were hospitalized in our hospital from March 2017 
to December 2017 are clinically diagnosed and histopathologically confirmed to meet the relevant diagnostic criteria. There are 46 males and 34 females with an average age of (57.5 \pm 5.5$)$. The patients are randomly divided into control group and treatment group, 44 cases in each group. In the

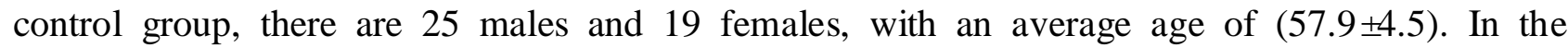
treatment group, there are 21 males and 15 females, with an average age of $(55.1 \pm 5.8)$. All the patients are approved by the ethics committee of the hospital and sign the informed consent form. There is no statistically significant difference between the two groups in the general data (gender, age and disease severity) ( $\mathrm{P}>0.05)$. The comparability between groups is comparable.

\section{Method ${ }^{[5-7]}$}

To establish a WeChat public platform for the patients in the treatment group: After applying via the Internet, set up user name and account number of WeChat public platform. Use the generated specific QR code as the head of the public platform number, and establish a dedicated WeChat public platform for hepatobiliary surgery. Its back-end materials mainly include the introduction of rules and regulations, education on health knowledge, and texts which can encourage patients to maintain a good attitude. The data forms include text, pictures, expressions, videos, etc. According to the characteristics of the department and patients' knowledge needs, the hospital will eventually provide guidance and maintenance from two aspects, which are in-hospital guidance and out-of-hospital care. The main framework and main interface of WeChat public platform include admission guidance, the guidance before and after surgery, healthy diet, discharge guidance, various inspections and other health education knowledge communicate with the culture, style and difficult issues of the department. After patients enter the hospital, they can search for WeChat public platform user name or scan to generate the two-dimensional code to WeChat groups according to their own wishes. If there are problems, they can directly send relevant information to WeChat public platform, and then the administrator will perform, reply and answer one by one according to the situation.

\section{Evaluation Index}

The state of mentality is measured by SAS to evaluate the degree of anxiety before and after the patients' care. Each score is added up to a total score. After the total score is $\times 1.25$, a standard score is obtained. The higher the score is, the more severe the degree of anxiety of patients will be. When the patient's overall score is less than 40 points, it indicates that his mood is relatively stable; when the overall score is greater than or equal to 40 points, the patient's mood anxiety is indicated. GSES is used to evaluate the quality of life of liver cancer patients. QOL is used to evaluate PF, RF, BP and SF. The total score is 100 points. The higher the score is, the better the quality of life will be. According to the criteria of depressive effect, the score of HAMD, the effect is divided into four grades, which are recovery, significant effect, effective and ineffective. Recovery: Symptoms disappear after treatment. The efficacy index of the test is $>75 \%$. Significant effect: Symptoms significantly reduce. The efficacy index is in the range of 50-75\%. Effective: Symptoms partially disappear. The efficacy index is in the range of $25-50 \%$. Ineffective: There is no significant change or worsening of symptoms after treatment, and the efficacy index is less than $25 \%$.

\section{Analytical method}

The results obtained after treatment of the two groups of different treatment methods are compared and analyzed using SPSS 14.0 statistical software. The t-test method is applied to measure data using mean \pm standard deviation $(\mathrm{x} \pm \mathrm{s})$. The count data is tested using the $\mathrm{X}^{2}$ test rate $(\%)$. There is significant difference between groups if $\mathrm{P}<0.05$.

\section{Results}

\section{HAMD score results}

From the results, it can be seen that HAMD score in the treatment group at different time points after the treatment is significantly lower than that before the treatment. Compared with the control group, there is a significant difference between the groups $(\mathrm{P}<0.05)$, as shown in Table 1. 
Tab. 1 HAMD scores in both groups

\begin{tabular}{cccccc}
\hline & & \multicolumn{2}{c}{$\begin{array}{c}\text { Before } \\
\text { treatment }\end{array}$} & \multicolumn{3}{c}{ After treatment } \\
\cline { 3 - 6 } Group & $\mathrm{n}$ & HAMD score & $\begin{array}{c}1 \text { month after } \\
\text { treatment }\end{array}$ & $\begin{array}{c}2 \text { months } \\
\text { after } \\
\text { treatment }\end{array}$ & $\begin{array}{c}3 \text { months } \\
\text { after } \\
\text { treatment }\end{array}$ \\
\hline $\begin{array}{c}\text { Treatment } \\
\text { group }\end{array}$ & 44 & $27.57 \pm 2.14$ & $20.56 \pm 2.44$ & $15.57 \pm 1.86$ & $10.35 \pm 1.49$ \\
Control group & 44 & $27.33 \pm 2.22$ & $25.58 \pm 2.37$ & $22.32 \pm 2.05$ & $20.0 \pm 1.86$ \\
\hline
\end{tabular}

Patients' Quality of Life Score in Both Groups

Compared with the control group, the score of PF, MH, BP and SF in the quality of life of the patients after treatment in the treatment group is significantly improved, and the difference between the groups is significant $(\mathrm{P}<0.05)$, as shown in Table 2 .

Tab. 2 Patients' quality of life scores in both groups

\begin{tabular}{ccccc}
\hline Group & PF & MH & BP & SF \\
\hline $\begin{array}{c}\text { Treatment group } \\
(\mathrm{n}=44)\end{array}$ & $90.8 \pm 4.3$ & $75.4 \pm 2.8$ & $92.0 \pm 4.4$ & $88.0 \pm 3.9$ \\
Control group $(\mathrm{n}=44)$ & $77.1 \pm 6.6$ & $40.7 \pm 3.6$ & $54.1 \pm 5.7$ & $63.6 \pm 5.8$ \\
$\mathrm{P}$ & $<0.05$ & $<0.05$ & $<0.05$ & $<0.05$ \\
\hline
\end{tabular}

\section{Compare SAS Index Score between Two Groups of Patients}

It can be known by measuring the SAS index score of the patients of the treatment group and the control group, compared with the control group, SAS anxiety score of the patients of the treatment group significantly decreases and there is a significant difference between the groups $(\mathrm{P}<0.05)$. The results show that the treatment group have a clinical effect of health education, which is obviously better than the control group, see Table 3 for details.

Tab. 3 Comparison of SAS index values between two groups of patients (point,

$\bar{x} \pm s)$

\begin{tabular}{cccc}
\hline Group & $\mathrm{n}$ & $\begin{array}{c}\text { Before treatment SAS } \\
\text { score }\end{array}$ & After treatment SAS score \\
\hline $\begin{array}{c}\text { Treatment } \\
\text { group }\end{array}$ & 44 & $62.8 \pm 5.4$ & $20.11 \pm 5.78$ \\
Control group & 44 & $61.9 \pm 4.69$ & $38.45 \pm 6.50$ \\
\hline
\end{tabular}

\section{Compare the Satisfaction between Two Groups of Patients}

The satisfaction results of psychological nursing interventions of the patients of the two groups are shown in Table 2. Among the patients in the treatment group, 23 are satisfied with the nursing intervention, in which there are 6 patients who are satisfied with nursing care, and the satisfaction rate is $96.55 \%$; 12 patients are satisfied in the control group, in which there are 5 patients who are satisfied with nursing care, and the satisfaction is only $51.72 \%$. The satisfaction of the recovery nursing group is increased by $40 \%$. The degree of satisfaction between the groups is large with significant differences between the groups $(\mathrm{P}<0.05)$.

Tab. 4 Comparison of satisfaction between two groups of patients $(n)$

\begin{tabular}{cccccc}
\hline Group & $\mathrm{n}$ & $\begin{array}{c}\text { Satisfie } \\
\mathrm{d}\end{array}$ & $\begin{array}{c}\text { Genera } \\
1\end{array}$ & Unsatisfied & $\begin{array}{c}\text { Satisfaction rate } \\
(\%)\end{array}$ \\
\hline Treatment group & 44 & 27 & 15 & 2 & 95.5 \\
Control group & 44 & 20 & 13 & 11 & 75.0 \\
\hline
\end{tabular}




\section{Conclusion}

Liver cancer is a common multiple malignant syndrome in clinical practice. It has the characteristics of serious illness, high incidence, rapid onset of acute polyposis and rapid progression of the disease. Currently, effective screening methods are not perfect because of high malignancy and rapid development. And the occurrence is more hidden, the patient has arrived at the advanced stage of cancer before seeing a doctor. The mortality rate is higher in all malignant tumors ${ }^{[8-9]}$.

It is caused by multiple factors, such as nausea, vomiting, edema and other clinical manifestations, and even threatens the lives of patients. Most of the symptoms are not obvious at the early stage, and attention cannot be paid to timely and effective treatment. The disease is prone to pathological changes. The etiology of the disease is often complex and diverse. At present, it is widely believed that the development of liver cancer is related to multi-gene mutation, multi-linkage, multi-phase evolution conditions. Cell signal transduction plays an important role in the regulation of cell growth, because changes in any component of its signaling pathway will affect the regulation of cell proliferation, apoptosis and carcinogenesis. High clinical morbidity and mortality, serious psychological and physical harm and serious impact on the quality of life of patients $^{[10-11]}$. Surgery is the main treatment measure. But it brings physical and mental pain to patients, which aggravates patients' psychological burden, contradicts their minds and even makes them give up lives. Researchers should actively take effective treatments for this disease in clinical practice. Therefore, it is imperative to further inquire into it, and the establishment of a comprehensive treatment plan is the key. Effective treatment of the disease is particularly important. If the condition is not completely or not timely controlled, it will result in mistreatment or complications. During the nursing process, a new care model is adopted to better provide patients with care measures in various ways. In order to actively cooperate with the doctors to improve their quality of life, they should be as careful as possible and implement specific details, which will help improve the treatment of patients, which will increase patients' compliance, satisfaction with care, reduce adverse effects and improve the quality of life of patients, and is conducive to achieve the best effect, reduce the degree of harm, and play a positive role in patient treatment.

This study shows that compared with the control group, the SAS score of the patients in the treatment group is significantly reduced, and the difference between the groups is significant $(\mathrm{P}<0.05)$. Compared with the control group, the anxiety score of the patients in the treatment group decrease significantly, and there is a significant difference between groups $(\mathrm{P}<0.05)$. In terms of quality of life, the scores of the indicators (PF, MH, BP, SF) in the treatment group are signif icantly improved and the results are better. The difference between the groups is significant $(\mathrm{P}<0.05)$. It shows that health education nursing intervention will help patients with liver cancer to reach a certain degree of recovery. In summary, the health education care model on WeChat public platform has a significant role in promoting the effectiveness of liver cancer patients and improving their quality of life.

In summary, the health education intervention of WeChat public platform can help patients recover their health, improve comfort and improve their prognosis. It can also increase patients' compliance with the treatment and increase their satisfaction with WeChat health education and improve their quality of life, which can show the superiority of the treatment. It is more helpful to control the complications, is of certain guiding value in the clinical, and can provide an ideal intervention method for clinical treatment, which is worthy of clinical implementation.

\section{References}

[1] Chen Junhua, Feng Guoju, Ya Chunmei. The effect of nursing intervention on the quality of life of liver cancer patients after interventional therapy [J]. Hebei Medical, 2009, 31 (6): 749-750.

[2] Zheng Ning, Chu Wenxia. Application of Health Education in Clinical Nursing [J]. Occupation and Health, 2005, 21(10):1656.

[3] Chen Shaobo, Lu Aiping. Stroke patient health education [J]. Nursing Research, 2007, 
21(6):1618.

[4] Wang Yang. Hospital patient follow-up tracking system design and implementation [J]. Science and Technology Innovation Review, 2009(28):240-242.

[5] Zhao Fuyun, Xu Congfang. Interventional strategies for quality of life of cancer patients [J]. International Journal of Nursing, 2009, 28(1): 66.

[6] Cheng Yanshuang, Zhang Yameng, Ma Yanyong. Effect of health education based on WeChat public platform on quality of life of liver cancer patients[J].nursing research.2017,31(7):848-852.

[7] Liu Jingli, Qiu Hong, Liu Dongbo, et al. Incidence of anxiety and depression in cancer patients and analysis of influencing factors[J].Drug Evaluation,2012,9(3):24-28.

[8] Li Shuxing, Zhang Min, Chen Changxiang, et al. Investigation and analysis of the quality of life of the elderly with common chronic diseases[J]. Chinese Journal of Gerontology, 2014 (8): 2240-2243.

[9] Jia Lina, Zhuang Hailin, Chen Yue, et al. Analysis of quality of life and influencing factors of elderly patients with chronic diseases in the home[J]. Chinese Journal of Geriatrics, 2013, 32 (12): 1361-1364.

[10] Zhang Xiaozhu. Effects of Family Function and Social Support on Drug Compliance in Patients with Hypertension[J]. Journal of Nursing Management, 2014, 14 (3 ): 173-175.

[11] Ma Jianqing. Comprehensive interventions on the impact of self-efficacy on the self-efficacy of patients with chronic diseases and the function of their families [J].Syndrome of Chronic Diseases.2016,17(6):700-704. 